\title{
Freshwater Amphipod Specimen Preparation for TEM
}

\author{
Suzanne S. Tyler*, and Gretchen A. Messick \\ *NOAA, National Ocean Service, Cooperative Oxford Lab, 904 S. Morris Street, Oxford, MD \\ 21654 \\ NOAA, National Ocean Service, Cooperative Oxford Lab, 904 S. Morris Street, Oxford, MD \\ 21654
}

Amphipods of Diporeia spp. have been declining in numbers during the last decade in the Great Lakes. Fish species that relied heavily on Diporeia spp. are declining in abundance or shifting feeding patterns. To help identify the problem, a histological survey of the population was performed using light microscopy and transmission electron microscopy (TEM) techniques. Light microscopy revealed numerous diseases of interest that could be linked to the population decline [1]. To better identify the disease agents and elucidate their morphology, routine TEM was performed on the amphipods. While there is much written regarding the biological processing of specimens for TEM, we describe techniques specific for TEM processing of amphipods.

Amphipods were collected and stored in $2.5 \%$ glutaraldehyde in $0.1 \mathrm{M}$ sodium cacodylate buffer $\mathrm{pH} 7.4$ [2]. After 12-16 hours, tissues were transferred to $0.1 \mathrm{M}$ sodium cacodylate buffer at $4^{\circ} \mathrm{C}$ until processing. Each animal was bisected longitudinally to retain the same organ systems; one half was used for paraffin embedding, the other half saved for epoxy embedding at a later time. The appendages were used as a guideline for making the longitudinal cut through the ventral surface of the animal.

To minimize any tissue distortion, a 2 hour schedule without vacuum was used for processing the tissues for light microscopy. To save both time and chemicals, all four amphipods from the same cassette were embedded together, and oriented in the same position. The position of each amphipod was noted so it could be identified for future reference. Samples were sectioned to a thickness of 3- to 4- $\mu \mathrm{m}$. The resulting ribbon was mounted on a poly-L-lysine-coated slide and stained with Mayer's hematoxylin and eosin.

Once the pathologist determined the need to perform TEM, the "sister sample" was retrieved from the buffer solution and postfixed with buffered 1\% osmium tetroxide for 1 hour. Specimens were dehydrated through a graded series of ethanol solutions, and then cleared with a graded series of propylene oxide. Tissues were infiltrated with Spurr's epoxy resin and embedded using a flat mold. Thick sections were obtained and stained with $1 \%$ toluidine blue to confirm the presence of the organism. Final thin sections were cut using a diamond knife, placed on a 200 mesh copper grid, and stained using routine uranyl acetate lead staining procedures.

Common techniques utilized in clinical histology can also be applied to unique studies involving freshwater amphipods. 


\section{References}

[1] G. A. Messick et al., Dis Aquatic Org 59 (2004) 159-170.

[2] M.A. Hayat, Principles and Techniques of Electron microscopy, Van Nostrand, Reinhold, 1970.

[3] A. A. Anderson, Chlamydiosis. In: A Laboratory Manual for the Isolation and Identification of Avian Pathogens, $4^{\text {th }}$ ed., Kendall/Hunt, Dubuque, 1998.

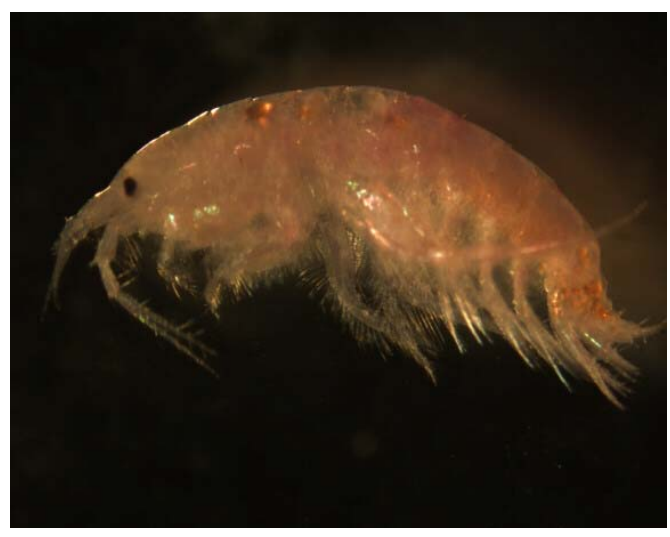

Freshwater amphipod Diporeia spp.

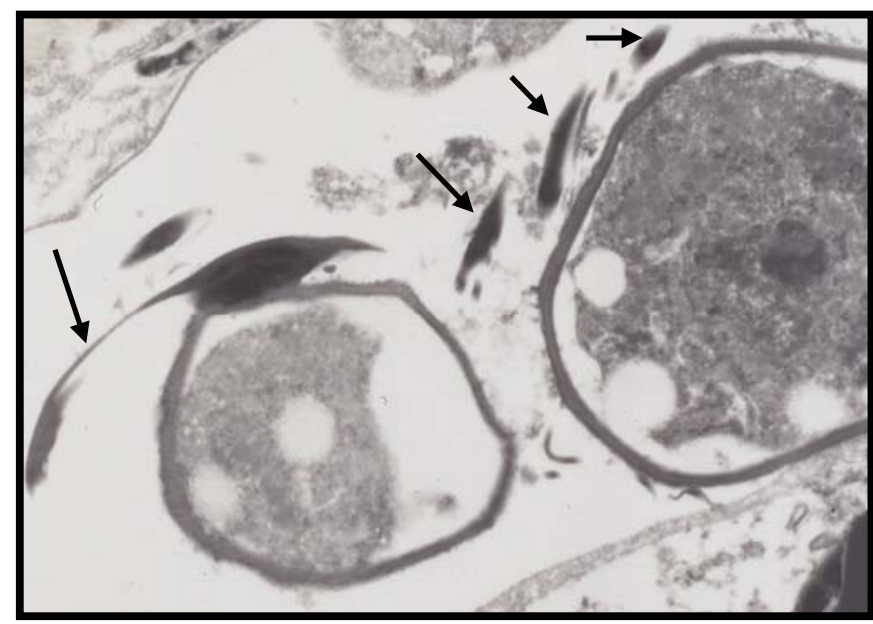

Haplosporidian in amphipod host showing structures that may be used for species identification (original mag 10k).

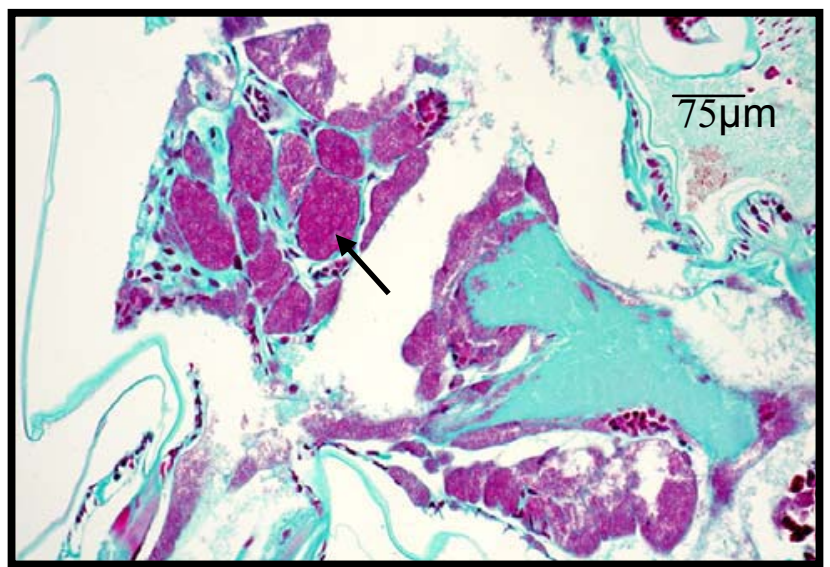

Light micrograph of Pierce Van der Kamp stain for rickettsia [3].

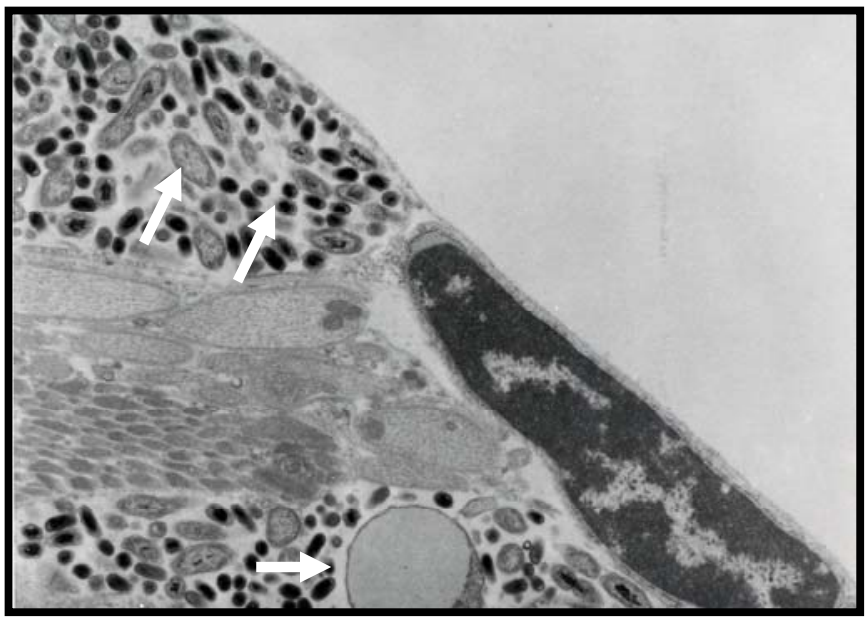

Different morphologies of rickettsia-like organism found in amphipod host tissue (original mag 8k). 